\title{
An Iterative Learning Control Design Method for Nonlinear Discrete-Time Systems with Unknown Iteration-Varying Parameters and Control Direction
}

\author{
Duo Zhao and Yong Yang \\ School of Electrical Engineering, Southwest Jiaotong University, Chengdu 610031, China \\ Correspondence should be addressed to Yong Yang; dr_yongyang@163.com
}

Received 16 May 2016; Revised 30 August 2016; Accepted 1 September 2016

Academic Editor: Xuejun Xie

Copyright @ 2016 D. Zhao and Y. Yang. This is an open access article distributed under the Creative Commons Attribution License, which permits unrestricted use, distribution, and reproduction in any medium, provided the original work is properly cited.

An iterative learning control (ILC) scheme is designed for a class of nonlinear discrete-time dynamical systems with unknown iteration-varying parameters and control direction. The iteration-varying parameters are described by a high-order internal model (HOIM) such that the unknown parameters in the current iteration are a linear combination of the counterparts in the previous certain iterations. Under the framework of ILC, the learning convergence condition is derived through rigorous analysis. It is shown that the adaptive ILC law can achieve perfect tracking of system state in presence of iteration-varying parameters and unknown control direction. The effectiveness of the proposed control scheme is verified by simulations.

\section{Introduction}

Iterative learning control (ILC) is an effective control method in improving the transient response and tracking performance of controlled system when the control task is performed repeatedly in a finite time interval [1]. The main idea of ILC is to modify the control input profile by using the deviation of the system output and the desired trajectory so that the track performance can be improved continuously along the iteration axis. Recently, more and more attentions have been put towards ILC design under more general problem settings as well as application of the well-established ILC schemes to industrial and engineering processes [2-8].

Traditional framework of ILC design needs the strict repeatability of processes, which however is hard to be met in practice. As a result, ILC design with iteration-varying factors is a problem of considerable importance in both theory and practical applications [9]. For example, the iteration-varying initial state $[10,11]$, reference $[12,13]$, and disturbances $[14$, 15] have been frequently encountered. In practice, along the iterative axis, these factors can be described by high-order internal models (HOIMs) [16]; that is, the iteration-varying factors in the current iteration are linear combinations of the counterparts in the previous certain iterations [17]. It is worth noticing that although HOIM information has been considered to expedite the learning convergence of ILC in $[9,16,17]$, there have been no works addressing ILC design of nonlinear discrete-time systems with iteration-varying HOIM-type uncertainties.

The main contribution of the paper lies in the fact that HOIM-based ILC scheme is proposed for a class of nonlinear discrete-time systems with unknown control direction [1821]. The learning convergence condition is derived through rigorous analysis. It is shown that the proposed adaptive ILC law can achieve perfect tracking of system state in presence of iteration-varying parameters and unknown control direction. The paper is organized as follows. In Section 2, the problem formulation is given. In Section 3, an adaptive ILC scheme is proposed to achieve perfect tracking. of system output. In Section 4, the learning convergence of the proposed control scheme is addressed rigorously. In Section 5, the effectiveness of the proposed control scheme is verified by simulations. Section 6 concludes the work. 


\section{Problem Formulation}

Consider the following discrete-time system:

$$
\begin{aligned}
& x_{i, k}(t+1)=x_{i+1, k}(t), \quad i=1, \ldots, n-1, \\
& x_{n, k}(t+1)=\theta_{k}(t) f\left(\mathbf{x}_{k}(t), t\right)+b(t) u_{k}(t),
\end{aligned}
$$

where $x_{i, k}(t) \in \mathscr{R}$ denotes the $i$ th state variable at the $t$ th time instant of the $k$ th iteration, $t \in[0,1, \ldots, T], \mathbf{x}_{k} \triangleq$ $\left[x_{1, k}, \ldots, x_{n, k}\right]^{T} \in \mathscr{R}^{n}$ is the state vector with random initial condition $\mathbf{x}_{k}(0)$ in each iteration $k, u_{k}(t) \in \mathscr{R}$ is the system input, $\theta_{k}(t)$ is an unknown iteration-varying bounded parameter, $f\left(\mathbf{x}_{k}(t), t\right)$ is a known nonlinear regressor function, and $b(t) \geq b_{\min }>0, \forall t$ is the unknown time-varying input gain function. The case that $b(t)<0, \forall t$, can be considered similarly by redefining the input profile.

Define the desired trajectory as $\mathbf{x}_{d}(t)=\left[x_{1, d}(t), \ldots\right.$, $\left.x_{n, d}(t)\right]^{T}, t \in[0,1, \ldots, T]$, and assume that $\mathbf{x}_{d}(t)$ is bounded and generated by the following reference model:

$$
\begin{aligned}
& x_{i, d}(t+1)=x_{i+1, d}(t), \quad i=1, \ldots, n-1, \\
& x_{n, d}(t+1)=g\left(\mathbf{x}_{d}(t), t\right),
\end{aligned}
$$

where the function $g$ is continuous with respect to its arguments.

Then, the tracking error at the $k$ th iteration is $e_{i, k}(t) \triangleq$ $x_{i, k}(t)-x_{i, d}(t)$. From (1) and (2), it follows that

$$
\begin{aligned}
e_{i, k}(t+1)= & e_{i+1, k}(t), \quad i=1, \ldots, n-1, \\
e_{n, k}(t+1)= & \theta_{k}(t) f\left(\mathbf{x}_{k}(t), t\right)+b(t) u_{k}(t) \\
& -g\left(\mathbf{x}_{d}(t), t\right) .
\end{aligned}
$$

The control target is to find a sequence of system input $u_{k}(t)$ so that the system state $\mathbf{x}_{k}(t)$ of (1) can converge to the desired trajectory $\mathbf{x}_{d}(t)$ asymptotically along the iteration axis.

We shall make some assumptions first.

Assumption 1 (see [16]). The iteration-varying parameter $\theta_{k}(t)$ satisfies

$$
\theta_{k}(t)=a_{1} \theta_{k-1}(t)+\cdots+a_{m} \theta_{k-m}(t),
$$

where $a_{1}, \ldots, a_{m}, m \geq 1$, are known constant parameters and the initial parameters $\theta_{0}(t), \ldots, \theta_{1-m}(t)$ are unknown functions that are linearly independent. In other words, $\theta_{k}(t)$ satisfies HOIM with order $m$.

Assumption 2. The nonlinear function $f\left(\mathbf{x}_{k}(t), t\right)$ satisfies the linear growth condition; that is, $\left|f\left(\mathbf{x}_{k}(t), t\right)\right| \leq c_{1}+c_{2}\left\|\mathbf{x}_{k}(t)\right\|$, where $c_{1}$ and $c_{2}$ are positive constants.

The following lemma will be used in deriving the learning convergence of the proposed control scheme.

Lemma 3 (the Key Technical Lemma [22]). If

$$
\lim _{t \rightarrow \infty} \frac{s^{2}(t)}{b_{1}(t)+b_{2}(t) \sigma^{T}(t) \sigma(t)}=0,
$$

where $b_{1}(t), b_{2}(t)$, and $s(t)$ are real scalar sequence and $\sigma(t)$ is a real vector sequence, and the following two conditions hold:

(1) uniform boundedness conditions $0<b_{1}(t)<K<\infty$ and $0<b_{2}(t)<K<\infty$ for all $t>0$;

(2) linear boundedness condition

$$
\|\sigma(t)\| \leq C_{1}+C_{2} \max _{0<\tau<t}|s(\tau)|,
$$

where $0<C_{1}<\infty$ and $0<C_{2}<\infty$, then we have

$$
\lim _{t \rightarrow \infty} s(t)=0
$$

and $\|\sigma(t)\|$ is bounded. $\|\cdot\|$ denotes the Euclid norm.

Define $\varphi_{j}(t)=\theta_{j-m}(t)$, where $j=1,2, \ldots, m$ and $\boldsymbol{\theta}_{k}^{*}(t) \triangleq$ $\left[\theta_{k-m+1}, \ldots, \theta_{k}(t)\right]^{T}$ with $\boldsymbol{\theta}_{0}^{*}(t)=\left[\varphi_{1}(t), \ldots, \varphi_{m}(t)\right]^{T}$. Then we can rewrite (4) as

$$
\boldsymbol{\theta}_{k+1}^{*}(t)=B \boldsymbol{\theta}_{k}^{*}(t)
$$

where

$$
B \triangleq\left(\begin{array}{cccccc}
0 & 1 & 0 & \cdots & 0 & 0 \\
0 & 0 & 1 & \cdots & 0 & 0 \\
\vdots & \vdots & \vdots & \ddots & \vdots & \vdots \\
0 & 0 & 0 & \cdots & 0 & 1 \\
a_{m} & a_{m-1} & a_{m-2} & \cdots & a_{2} & a_{1}
\end{array}\right) .
$$

Repeating (8), we obtain

$$
\boldsymbol{\theta}_{k}^{*}(t)=B \boldsymbol{\theta}_{k-1}^{*}(t)=\cdots=B^{k} \boldsymbol{\theta}_{0}^{*}(t) .
$$

Let $\beta_{k} \triangleq\left[\beta_{1, k}, \cdots, \beta_{m, k}\right]^{T}$ be the last row of the matrix $B^{k}$; it renders to

$$
\theta_{k}(t)=\beta_{1, k} \varphi_{1}(t)+\cdots+\beta_{m, k} \varphi_{m}(t)=\boldsymbol{\beta}_{k}^{T} \boldsymbol{\theta}_{0}^{*}(t) .
$$

Notice that $\beta_{1}=\left[a_{m}, \ldots, a_{1}\right]$. Owing to the boundedness of $\theta_{k}(t), \beta_{j, k}$ is also bounded; that is, there exists $\beta>0$ such that $\left|\beta_{j, k}\right| \leq \beta$ for $j=1,2, \ldots, m$ and $k=1,2, \ldots$. As such, the last equation of system (1) can be written in a more compact form:

$$
\begin{aligned}
x_{n, k}(t+1) & =\sum_{j=1}^{m} \varphi_{j}(t) \beta_{j, k} f\left(\mathbf{x}_{k}(t), t\right)+b(t) u_{k}(t) \\
& =\boldsymbol{\varphi}^{T}(t) \boldsymbol{\eta}_{k}(t)+b(t) u_{k}(t),
\end{aligned}
$$

where $\boldsymbol{\varphi}(t) \triangleq\left[\varphi_{1}(t), \ldots, \varphi_{m}(t)\right]^{T}$ and $\boldsymbol{\eta}_{k}(t) \triangleq\left[\beta_{1, k} f\left(\mathbf{x}_{k}(t)\right), \ldots\right.$, $\left.\beta_{m, k} f\left(\mathbf{x}_{k}(t)\right)\right]^{T}$.

Remark 4. From system (12), the estimation of the iterationvarying parameter $\theta_{k}(t)$ is transformed to that of the iteration-invariant parameter $\varphi(t)$. It implies that the parametric updating law and the control law can be, more conveniently, designed in the iteration domain. This is the main reason why the iteration-varying parameters satisfying HOIM can be addressed along the proposed way. 


\section{Controller Design}

In this section, by making full use of the HOIM information of the parametric uncertainties $\theta_{k}(t)$, an ILC controller is designed for the considered nonlinear discrete-time system (1). Notice that the dynamics of $x_{n, k}$ in (1) has been reformulated as (12), where the parametric uncertainties $\varphi(t)$ and $b(t)$ are iteration-invariant.

The control law is given as

$$
\begin{aligned}
u_{k}(t) & =\frac{-\sum_{j=1}^{m} \widehat{\varphi}_{j, k}(t) \beta_{j, k} f\left(\mathbf{x}_{k}(t)\right)+x_{n, d}(t+1)}{\operatorname{proj}\left(\widehat{b}_{k}(t)\right)} \\
& =\frac{-\widehat{\boldsymbol{\varphi}}_{k}^{T}(t) \boldsymbol{\eta}_{k}(t)+x_{n, d}(t+1)}{\operatorname{proj}\left(\widehat{b}_{k}(t)\right)}
\end{aligned}
$$

where $\widehat{\varphi}_{j, k}(t), j=1,2, \ldots, m$, and $\widehat{b}_{k}(t)$ are the estimates of $\varphi_{j}(t)$ and $b(t)$ at the $k$ th iteration, respectively, $\widehat{\varphi}_{k}(t) \triangleq$ $\left[\widehat{\varphi}_{1, k}(t), \ldots, \widehat{\varphi}_{m, k}(t)\right]^{T}$, and $\operatorname{proj}(\cdot)$ is a projection operator defined as [23]

$$
\operatorname{proj}\left(\widehat{b}_{k}(t)\right)= \begin{cases}\widehat{b}_{k}(t), & \text { if }\left|\widehat{b}_{k}(t)\right| \geq b_{\min }, \\ \widehat{b}_{k-1}(t), & \text { otherwise, }\end{cases}
$$

where $b_{\min }$ is the lower bound of the unknown control gain $b(t)$. By using the projection operator function, the possible singularity in (13) can be avoid. In fact, $\left|\operatorname{proj}\left(\widehat{b}_{k}(t)\right)\right| \geq b_{\min }$ if the initial condition is chosen as $\left|\widehat{b}_{0}(t)\right| \geq b_{\min }$.

Observing (13), we have

$$
\begin{aligned}
x_{n, d}(t+1)= & \sum_{j=1}^{m} \widehat{\varphi}_{j, k}(t) \beta_{j, k} f\left(\mathbf{x}_{k}(t)\right) \\
& +\operatorname{proj}\left(\widehat{b}_{k}(t)\right) u_{k}(t) .
\end{aligned}
$$

Then, by the definition of state tracking error $e_{i, k}(t)$,

$$
\begin{aligned}
e_{n, k}(t+1)= & \sum_{j=1}^{m} \varphi_{j}(t) \beta_{j, k} f\left(\mathbf{x}_{k}(t), t\right)+b(t) u_{k}(t) \\
& -\sum_{j=1}^{m} \widehat{\varphi}_{j, k}(t) \beta_{j, k} f\left(\mathbf{x}_{k}(t)\right) \\
& -\operatorname{proj}\left(\widehat{b}_{k}(t)\right) u_{k}(t) \\
= & {\left[\widehat{b}_{k}(t)-\operatorname{proj}\left(\widehat{b}_{k}(t)\right)\right] u_{k}(t) } \\
& -\widehat{\phi}_{k}^{T}(t) \xi_{k}(t)+\boldsymbol{\phi}^{T}(t) \boldsymbol{\xi}_{k}(t) \\
= & -\widetilde{\boldsymbol{\phi}}_{k}^{T}(t) \xi_{k}(t) \\
& +\left[\widehat{b}_{k}(t)-\operatorname{proj}\left(\widehat{b}_{k}(t)\right)\right] u_{k}(t),
\end{aligned}
$$

where $\widetilde{\boldsymbol{\phi}}_{k}(t) \triangleq\left[\widetilde{\varphi}_{1, k}(t), \ldots, \widetilde{\varphi}_{m, k}(t), \widetilde{b}_{k}(t)\right]^{T}, \widetilde{\varphi}_{j, k}(t) \triangleq \widehat{\varphi}_{j, k}(t)-$ $\varphi_{j}(t), \widetilde{b}_{k}(t) \triangleq \widehat{b}_{k}(t)-b(t), \boldsymbol{\phi}(t) \triangleq\left[\boldsymbol{\varphi}^{T}(t), b(t)\right]^{T}, \widehat{\boldsymbol{\phi}}_{k}(t) \triangleq\left[\widehat{\boldsymbol{\varphi}}_{k}^{T}(t)\right.$, $\left.\widehat{b}_{k}(t)\right]^{T}$, and $\boldsymbol{\xi}_{k}(t) \triangleq\left[\boldsymbol{\eta}_{k}^{T}(t), u_{k}(t)\right]^{T}$.
The parametric updating laws for $\widehat{\boldsymbol{\varphi}}_{k}(t)$ and $\widehat{b}_{k}(t)$ are directly given as follows:

$$
\begin{gathered}
\widehat{\varphi}_{j, k+1}(t)=\widehat{\varphi}_{j, k}(t)-\frac{\beta_{j, k} f\left(\mathbf{x}_{k}(t)\right)}{p+\boldsymbol{\xi}_{k}^{T}(t) \boldsymbol{\xi}_{k}(t)} \widetilde{\boldsymbol{\phi}}_{k}^{T}(t) \boldsymbol{\xi}_{k}(t), \\
j=1,2, \ldots, m, \\
\widehat{b}_{k+1}(t)=\widehat{b}_{k}(t)-\frac{u_{k}(t)}{p+\boldsymbol{\xi}_{k}^{T}(t) \boldsymbol{\xi}_{k}(t)} \widetilde{\boldsymbol{\phi}}_{k}^{T}(t) \boldsymbol{\xi}_{k}(t),
\end{gathered}
$$

where $p$ is a positive constant.

Remark 5. The ILC (13) with parameter updating laws (17) is an adaptive scheme, which is an extension of typical adaptive controller and repetitive control [24]. Moreover, this ILC borrows the idea of the HOIM-based ILC in $[9,16,17,25]$.

\section{Convergence Analysis}

In this section, the learning convergence of the proposed ILC scheme, that is, control law (13) and parametric updating laws (17), will be analyzed in a rigorous way.

Theorem 6. For nonlinear discrete-time system (1), under Assumptions 1 and 2, control law (13) and parametric updating laws (17) ensure that

(1) the parametric estimation $\widehat{\phi}_{k}(t), t \in[0, \ldots, T]$ is always bounded for all iterations,

(2) the tracking errors $e_{i, k}(t), \forall t \in[n-i+1, \ldots, T], i=$ $1, \ldots, n$, will converge to zero asymptotically as $k \rightarrow$ $\infty$.

Proof. The whole proof is divided into two parts. Part 1 derives the boundedness of $\widehat{\phi}_{k}(t)$, and Part 2 addresses the asymptotical convergence of $e_{i, k}(t)$.

Part 1 (the boundedness of $\widehat{\phi}_{k}(t)$ ). Define the composite energy function at the $k$ th iteration as

$$
V_{k}(t)=\widetilde{\phi}_{k}^{T}(t) \widetilde{\phi}_{k}(t)=\sum_{j=1}^{m} \widetilde{\varphi}_{j, k}^{2}(t)+\widetilde{b}_{k}^{2}(t),
$$

whose difference in two consecutive iterations is

$$
\Delta V_{k+1} \triangleq V_{k+1}-V_{k}=\sum_{j=1}^{m}\left(\widetilde{\varphi}_{j, k+1}^{2}-\widetilde{\varphi}_{j, k}^{2}\right)+\widetilde{b}_{k+1}^{2}-\widetilde{b}_{k}^{2} .
$$

For the first part of the right hand side of (19), applying the learning laws (17) yields

$$
\begin{aligned}
\sum_{j=1}^{m}\left(\widetilde{\varphi}_{j, k+1}^{2}-\widetilde{\varphi}_{j, k}^{2}\right)= & \sum_{j=1}^{m}\left(\varphi_{j}-\widehat{\varphi}_{j, k+1}\right)^{2} \\
& -\sum_{j=1}^{m}\left(\varphi_{j}-\widehat{\varphi}_{j, k}\right)^{2} \\
= & 2 \sum_{j=1}^{m}\left(\widehat{\varphi}_{j, k}-\varphi_{j}\right)\left(\widehat{\varphi}_{j, k+1}-\widehat{\varphi}_{j, k}\right)
\end{aligned}
$$




$$
\begin{gathered}
+\sum_{j=1}^{m}\left(\widehat{\varphi}_{j, k+1}-\widehat{\varphi}_{j, k}\right)^{2} \\
=-2 \sum_{j=1}^{m} \frac{\widetilde{\varphi}_{j, k} \beta_{j, k} f\left(\mathbf{x}_{k}\right)}{p+\boldsymbol{\xi}_{k}^{T} \boldsymbol{\xi}_{k}} \widetilde{\boldsymbol{\phi}}_{k}^{T} \boldsymbol{\xi}_{k} \\
+\sum_{j=1}^{m} \frac{\left(\beta_{j, k} f\left(\mathbf{x}_{k}\right)\right)^{2}}{\left(p+\boldsymbol{\xi}_{k}^{T} \boldsymbol{\xi}_{k}\right)^{2}}\left(\widetilde{\boldsymbol{\phi}}_{k}^{T} \boldsymbol{\xi}_{k}\right)^{2} .
\end{gathered}
$$

For the second part of the right hand side of (19), similar procedure leads to

$$
\begin{aligned}
\widetilde{b}_{k+1}^{2}-\widetilde{b}_{k}^{2} & =2\left(\widehat{b}_{k}-b\right)\left(\widehat{b}_{k+1}-\widehat{b}_{k}\right)+\left(\widehat{b}_{k+1}-\widehat{b}_{k}\right)^{2} \\
& =-2 \frac{\widetilde{b}_{k} u_{k}}{p+\boldsymbol{\xi}_{k}^{T} \xi_{k}} \widetilde{\boldsymbol{\phi}}_{k}^{T} \boldsymbol{\xi}_{k}+\frac{u_{k}^{2}\left(\widetilde{\boldsymbol{\phi}}_{k}^{T} \boldsymbol{\xi}_{k}\right)^{2}}{\left(p+\boldsymbol{\xi}_{k}^{T} \boldsymbol{\xi}_{k}\right)^{2}} .
\end{aligned}
$$

Now, substituting (20) and (21) into (19) renders to

$$
\begin{aligned}
\Delta V_{k+1} & =-2 \frac{\left(\tilde{\boldsymbol{\phi}}_{k}^{T} \xi_{k}\right)^{2}}{p+\boldsymbol{\xi}_{k}^{T} \xi_{k}}+\frac{\boldsymbol{\xi}_{k}^{T} \xi_{k}\left(\tilde{\boldsymbol{\phi}}_{k}^{T} \boldsymbol{\xi}_{k}\right)^{2}}{\left(p+\boldsymbol{\xi}_{k}^{T} \xi_{k}\right)^{2}} \\
& =\left(-2+\frac{\boldsymbol{\xi}_{k}^{T} \xi_{k}}{p+\boldsymbol{\xi}_{k}^{T} \xi_{k}}\right) \frac{\left(\tilde{\boldsymbol{\phi}}_{k}^{T} \xi_{k}\right)^{2}}{p+\boldsymbol{\xi}_{k}^{T} \xi_{k}} \leq-\frac{\left(\tilde{\boldsymbol{\phi}}_{k}^{T} \xi_{k}\right)^{2}}{p+\xi_{k}^{T} \xi_{k}} \\
& \leq 0,
\end{aligned}
$$

implying that

$$
\tilde{\boldsymbol{\phi}}_{k+1}^{T}(t) \widetilde{\boldsymbol{\phi}}_{k+1}(t) \leq \widetilde{\boldsymbol{\phi}}_{k}^{T}(t) \widetilde{\boldsymbol{\phi}}_{k}(t) \leq \widetilde{\boldsymbol{\phi}}_{0}^{T}(t) \widetilde{\boldsymbol{\phi}}_{0}(t)
$$

or equivalently

$$
\left\|\widetilde{\phi}_{k+1}(t)\right\|^{2} \leq\left\|\widetilde{\phi}_{k}(t)\right\|^{2} \leq\left\|\widetilde{\phi}_{0}(t)\right\|^{2} .
$$

Considering the boundedness of $\varphi_{j}(t), b(t), \widehat{\varphi}_{j, 0}(t)$, and $\widehat{b}_{0}(t)$, it follows that $\left\|\widetilde{\phi}_{k}(t)\right\|$ is bounded, and therefore $\widehat{\phi}_{k}(t)$ is bounded, $\forall t \in[0,1, \ldots, T], k=1,2, \ldots$

Part 2 (the asymptotical convergence of $e_{i, k}(t)$ ). Our idea is to first prove the asymptotical convergence of $e_{n, k}(t)$, and then the asymptotical convergence of $e_{i, k}(t), i \leq n-1$, can be obtained immediately by the canonical form of the system.

On the one hand, we have from (22)

$$
V_{k+1}(t) \leq V_{0}(t)-\sum_{l=1}^{k} \frac{\left(\tilde{\phi}_{l}^{T} \xi_{l}\right)^{2}}{p+\xi_{l}^{T} \xi_{l}} .
$$

Since $V_{0}(t)$ is bounded and $V_{k+1}(t) \geq 0$, it is clear to see that

$$
\lim _{k \rightarrow \infty} \frac{\left(\widetilde{\boldsymbol{\phi}}_{k}^{T} \boldsymbol{\xi}_{k}\right)^{2}}{p+\boldsymbol{\xi}_{k}^{T} \xi_{k}}=0 .
$$

On the other hand, noticing the learning laws (17) and once again, we have

$$
\widehat{\phi}_{k+1}(t)=\widehat{\phi}_{k}(t)-\frac{\xi_{k}(t)}{p+\xi_{k}^{T}(t) \xi_{k}(t)} \widetilde{\phi}_{k}^{T}(t) \xi_{k}(t),
$$

yielding

$$
\begin{aligned}
\left\|\widehat{\boldsymbol{\phi}}_{k+1}-\widehat{\boldsymbol{\phi}}_{k}\right\|^{2} & =\frac{\xi_{k}^{T} \xi_{k}}{\left(p+\xi_{k}^{T} \xi_{k}\right)^{2}}\left(\widetilde{\boldsymbol{\phi}}_{k}^{T} \xi_{k}\right)^{2} \\
& \leq \frac{p+\xi_{k}^{T} \xi_{k}}{\left(p+\xi_{k}^{T} \xi_{k}\right)^{2}}\left(\widetilde{\boldsymbol{\phi}}_{k}^{T} \xi_{k}\right)^{2}=\frac{\left(\widetilde{\boldsymbol{\phi}}_{k}^{T} \xi_{k}\right)^{2}}{p+\boldsymbol{\xi}_{k}^{T} \xi_{k}} .
\end{aligned}
$$

Combining (26) and (28) leads to

$$
\lim _{k \rightarrow \infty}\left\|\widehat{\phi}_{k+1}-\widehat{\phi}_{k}\right\|=0 .
$$

Since $\widehat{\boldsymbol{\phi}}_{k}$ includes $\widehat{b}_{k}$ as the last entry, the following relationship is directly obtained from

$$
\lim _{k \rightarrow \infty}\left|\widehat{b}_{k}-\widehat{b}_{k-1}\right|=0 .
$$

By the definition of proj $\left(\widehat{b}_{k}\right),(30)$ renders to

$$
\lim _{k \rightarrow \infty}\left|\widehat{b}_{k}-\operatorname{proj}\left(\widehat{b}_{k}\right)\right|=0 .
$$

Further, observing $\boldsymbol{\xi}_{k}(t) \triangleq\left[\boldsymbol{\eta}_{k}^{T}(t), u_{k}(t)\right]^{T}$, it is clear that

$$
\left|u_{k}\right|=\sqrt{u_{k}^{2}} \leq \sqrt{p+\eta_{k}^{T} \boldsymbol{\eta}_{k}+u_{k}^{2}}=\sqrt{p+\xi_{k}^{T} \xi_{k}} .
$$

As a result,

$$
\frac{\left|\widehat{b}_{k}-\operatorname{proj}\left(\widehat{b}_{k}\right)\right|\left|u_{k}\right|}{\sqrt{p+\xi_{k}^{T} \xi_{k}}} \leq\left|\widehat{b}_{k}-\operatorname{proj}\left(\widehat{b}_{k}\right)\right| .
$$

Since the right hand side of (33) satisfies (31),

$$
\lim _{k \rightarrow \infty} \frac{\left|\widehat{b}_{k}-\operatorname{proj}\left(\widehat{b}_{k}\right)\right|\left|u_{k}\right|}{\sqrt{p+\xi_{k}^{T} \xi_{k}}}=0 .
$$

Now, noticing the error dyanmics of $e_{n, k}(t+1)$, that is, (16), the relationships (27) and (35) render to

$$
\lim _{k \rightarrow \infty} \frac{e_{n, k}(t+1)}{\sqrt{p+\xi_{k}^{T}(t) \xi_{k}(t)}}=0 .
$$

In order to prove the asymptotical convergence of $e_{n, k}$ via Lemma 3, namely, the Key Technical Lemma, it suffices to prove

$$
\begin{aligned}
\left\|\xi_{k}(t)\right\| \leq C_{1}+C_{2} \max _{t^{\prime} \in[0, t-1]}\left|e_{n, k}\left(t^{\prime}+1\right)\right|, & \\
t & =[1, \ldots, T],
\end{aligned}
$$


where $C_{1}$ and $C_{2}$ are certain finite constants. This will be addressed in the following.

By the definition of $\boldsymbol{\xi}_{k}(t)$,

$$
\left\|\xi_{k}(t)\right\| \leq\left|u_{k}(t)\right|+\left\|\boldsymbol{\eta}_{k}(t)\right\| .
$$

First evaluate the upper bound of $\left|u_{k}(t)\right|$. From the expression of the proposed controller (13),

$$
\begin{aligned}
\left|u_{k}(t)\right|= & \frac{\left|-\widehat{\boldsymbol{\varphi}}_{k}^{T}(t) \boldsymbol{\eta}_{k}(t)+x_{n, d}(t+1)\right|}{\left|\operatorname{proj}\left(\widehat{b}_{k}(t)\right)\right|} \\
\leq & \left|\operatorname{proj}\left(b_{k}(t)\right)\right|^{-1}\left\|\widehat{\boldsymbol{\varphi}}_{k}(t)\right\|\left\|\boldsymbol{\eta}_{k}(t)\right\| \\
& +\left|\operatorname{proj}\left(b_{k}(t)\right)\right|^{-1}\left|x_{n, d}(t+1)\right| \\
\leq & n_{1}+n_{2}\left\|\boldsymbol{\eta}_{k}(t)\right\|,
\end{aligned}
$$

where $n_{1} \triangleq b_{\min }^{-1} \tau$ and $n_{2} \triangleq b_{\min }^{-1} \max _{t \in[0, T]}\left\|\widehat{\boldsymbol{\varphi}}_{k}(t)\right\|$, and $\tau \triangleq \max _{t \in[0, T]}\left\|\mathbf{x}_{d}(t)\right\|$. The relationship $\left|\operatorname{proj}\left(b_{k}(t)\right)\right| \geq b_{\min }$ is adopted in deriving (38).

Second, we evaluate the upper bound of $\left\|\boldsymbol{\eta}_{k}(t)\right\|$. By the definition of $\boldsymbol{\eta}_{k}(t)$, it follows that

$$
\left\|\boldsymbol{\eta}_{k}(t)\right\|=\sqrt{\beta_{1, k}^{2}+\cdots+\beta_{m, k}^{2}}\left|f\left(\mathbf{x}_{k}(t)\right)\right| .
$$

Notice that $\left\|\mathbf{x}_{k}(t)\right\| \leq\left\|\mathbf{e}_{k}(t)\right\|+\left\|\mathbf{x}_{d}(t)\right\| \leq\left\|\mathbf{e}_{k}(t)\right\|+\tau$. Then, by Assumption 2, namely, the linear growth condition for the nonlinear regressor $f\left(\mathbf{x}_{k}(t)\right)$, we have

$$
\left|f\left(\mathbf{x}_{k}(t)\right)\right| \leq c_{1}+c_{2}\left\|\mathbf{x}_{k}(t)\right\| \leq c_{1}+c_{2} \tau+c_{2}\left\|\mathbf{e}_{k}(t)\right\| .
$$

Combining (39) and (40) gives

$$
\left\|\boldsymbol{\eta}_{k}(t)\right\| \leq \sqrt{m} \beta\left(c_{1}+c_{2} \tau\right)+\sqrt{m} \beta c_{2}\left\|\mathbf{e}_{k}(t)\right\|,
$$

where $\beta$ is an upper bound of $\beta_{i, k}, i=1, \ldots, m$.

Combining (38) and (41) yields

$$
\begin{aligned}
\left\|\boldsymbol{\xi}_{k}(t)\right\| & \leq\left|u_{k}(t)\right|+\left\|\boldsymbol{\eta}_{k}(t)\right\| \leq n_{1}+\left(1+n_{2}\right)\left\|\boldsymbol{\eta}_{k}(t)\right\| \\
& \leq q_{1}+q_{2}\left\|\mathbf{e}_{k}(t)\right\|,
\end{aligned}
$$

where $q_{1}=n_{1}+\sqrt{m} \beta\left(1+n_{2}\right)\left(c_{1}+c_{2} \tau\right)$ and $q_{2}=\sqrt{m} \beta\left(1+n_{2}\right) c_{2}$.

Now, the remaining is to find the relationship between $\left\|\mathbf{e}_{k}(t)\right\|$ and the quantity $\max _{t^{\prime} \in[0, t-1]}\left|e_{n, k}\left(t^{\prime}+1\right)\right|$. Observing the state error dynamics (3),

$$
e_{1, k}(t)=e_{2, k}(t-1)=\cdots=e_{n, k}(t-n+1),
$$

implying

$$
\left|e_{i, k}(t)\right|=\left|e_{n, k}(t-n+i)\right| \leq \max _{t^{\prime} \in[0, t]}\left|e_{n, k}\left(t^{\prime}\right)\right| .
$$

As such, we obtain

$$
\left\|\mathbf{e}_{k}(t)\right\| \leq \sum_{i=1}^{n}\left|e_{i, k}(t)\right| \leq n \max _{t^{\prime} \in[0, t]}\left|e_{n, k}\left(t^{\prime}\right)\right|
$$

Hence, by substituting (45) into (42), we have

$$
\begin{gathered}
\left\|\boldsymbol{\xi}_{k}(t)\right\| \leq q_{1}+q_{2}\left\|\mathbf{e}_{k}(t)\right\| \leq q_{1}+q_{2} n \max _{t^{\prime} \in[0, t]}\left|e_{n, k}\left(t^{\prime}\right)\right| \\
\leq q_{1}+q_{2}\left(n\left|e_{n, k}(0)\right|+n \max _{t^{\prime} \in[0, t-1]}\left|e_{n, k}\left(t^{\prime}+1\right)\right|\right) \\
\leq C_{1}+C_{2} \max _{t^{\prime} \in[0, t-1]}\left|e_{n, k}\left(t^{\prime}+1\right)\right|, \quad t=1, \ldots, T,
\end{gathered}
$$

where $C_{1} \triangleq q_{1}+C_{2}\left|e_{n, k}(0)\right|$ and $C_{2} \triangleq q_{2} n$.

At last, according to (43), the asymptotical convergence of $e_{n, k}(t), \forall t \in[1, \ldots, T]$, guarantees the asymptotical convergence of $e_{i, k}(t), \forall t \in[n-i+1, \ldots, T], i=1, \ldots, n-1$. The proof is complete.

Remark 7. The learning convergence of the proposed ILC scheme, that is, control law (13) and parametric updating laws (17), is proved rigorously for any random bounded initial states. In other words, the perfect tracking can be achieved for any random bounded initial conditions. The main reason is that the desired states at $t=1,2, \ldots, T$ of system (2) are directly utilized to regulate control input (13) and the effect of the state at $t=0$ can be ignored. In order to achieve perfect tracking, traditional ILC schemes restrict the initial states to be identical or convergent [9-11]. Hence, the efficiency in dealing with any random initial conditions is another contribution of our paper.

\section{Simulation Example}

Consider the following system:

$$
\begin{aligned}
& x_{1, k}(t+1)=x_{2, k}(t), \\
& x_{2, k}(t+1) \\
& =\theta_{k}(t)\left(\sin \left(\frac{\pi x_{1, k}(t)}{20}\right)+\cos \left(\frac{\pi x_{2, k}(t)}{30}\right)\right) \\
& \quad+b(t) u_{k}(t),
\end{aligned}
$$

where $b(t)=3+\sin (t)$ is the unknown time-varying control direction, $\theta_{k}(t)=-2 \cos (0.4) \theta_{k-1}(t)-\theta_{k-2}(t), \theta_{-1}(t)=$ $0.08 \cos (\pi t / 10)$, and $\theta_{0}(t)=-0.96 \sin (1.2 t)+0.8 \cos (2 t)$. The desired trajectories of the system are given by (2) with $g\left(\mathbf{x}_{d}(t), t\right)=1+0.5 e^{1-\cos (0.025 \pi t)}$. For demonstration, we set $T=200, \widehat{\varphi}_{1,0}(t)=\widehat{\varphi}_{2,0}(t)=0, \widehat{b}_{0}(t)=1$, and $b_{\min }=0.1$. In addition, the random initial condition of the system state, $\mathbf{x}_{k}(0)$, is shown in Figure 1.

The tracking performance is shown in Figures 2 and 3. More clearly, Figure 2 gives the maximum tracking error of $x_{2, k}(t), t \in[1,200]$, along the iteration axis. It can be seen that the tracking error is decreased significantly in 10 iterations and becomes invisible after 50 iterations. For illustration, the state profile of the system in the 70th iteration and its desired trajectory are given simultaneously in Figure 3. All these simulation results verify the effectiveness of the proposed ILC scheme. 

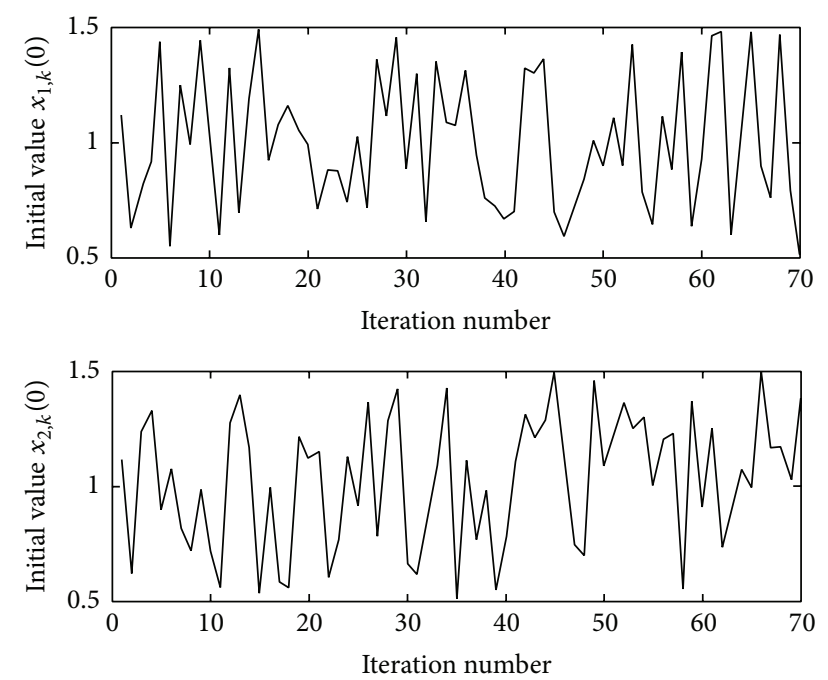

FIgURE 1: The random initial condition of the system state, $\mathbf{x}_{k}(0)$, versus the iteration number.

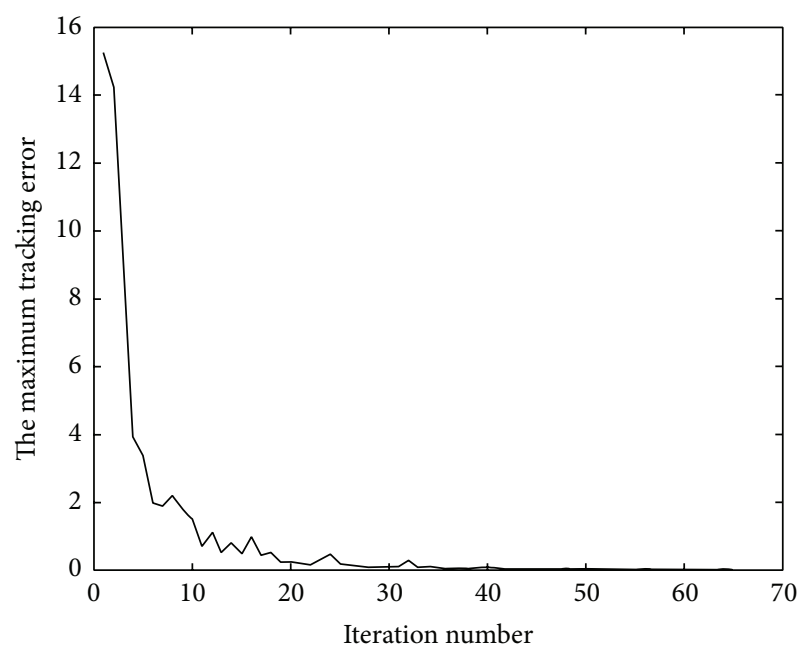

FIgURE 2: The profile of maximum tracking error of $x_{2, k}(t), t \in$ $[1,200]$, in the iteration domain.

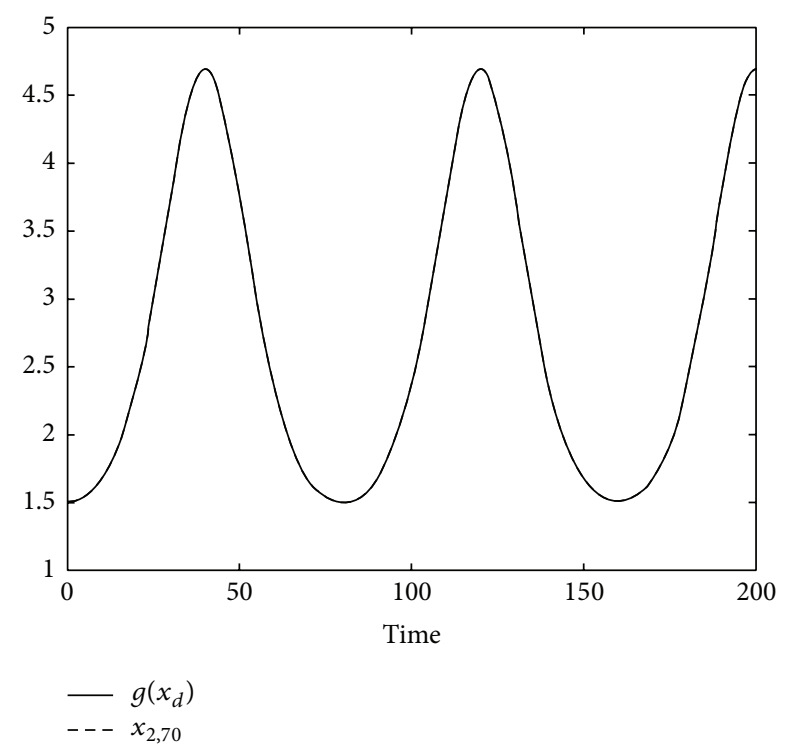

FIgURE 3: The state tracking profile, $x_{2,70}(t), t \in[1,200]$, and its desired trajectory.

\section{Conclusions}

In this paper, an iterative learning control scheme is presented for a class of nonlinear discrete-time systems with unknown iteration-varying parameters and unknown control direction, where the unknown iteration-varying parameters are assumed to satisfy a structure of high-order internal model (HOIM). By making full use of the information embedded in the HOIM, two efficient parametric updating laws are proposed to learn the system uncertainties. The learning convergence of the proposed control scheme is ensured through rigorous analysis. Our next research phase is to exploit the ILC design for systems with HOIM-type uncertainties but without linear growth conditions, as well as its applications.

\section{Competing Interests}

The authors declare that they have no competing interests.

\section{References}

[1] H.-S. Ahn, Y. Chen, and K. L. Moore, "Iterative learning control: brief survey and categorization," IEEE Transaction on Systems,Man, and Cybernetics-Part C: Application and Reviews, vol. 37, no. 6, pp. 1009-1121, 2007.

[2] S. S. Saab, "On the P-type learning control," IEEE Transaction on Automatic Control, vol. 39, no. 11, pp. 2298-2302, 1994.

[3] K.-H. Park, "An average operator-based PD-type iterative learning control for variable initial state error," IEEE Transaction on Automatic Control, vol. 50, no. 6, pp. 865-869, 2005.

[4] Z. Geng, R. Carroll, and J. Xie, "Two-dimensional model and algorithm analysis for a class of iterative learning control systems," International Journal of Control, vol. 52, no. 4, pp. 833$862,1990$.

[5] J.-X. Xu and Y. Tan, "A composite energy function-based learning control approach for nonlinear systems with timevarying parametric uncertainties," IEEE Transactions on Automatic Control, vol. 47, no. 11, pp. 1940-1945, 2002.

[6] R. Marino and P. Tomei, "An iterative learning control for a class of partially feedback linearizable systems," IEEE Transaction on Automatic Control, vol. 54, no. 8, pp. 1991-1996, 2009.

[7] D. Huang, V. Venkataramanan, J.-X. Xu, and T. C. T. Huynh, "Contact-induced vibration in dual-stage hard disk drive servo systems and its compensator design," IEEE Transactions on Industrial Electronics, vol. 61, no. 8, pp. 4052-4060, 2014.

[8] D. Huang, J.-X. Xu, V. Venkataramanan, and T. C. T. Huynh, "High-performance tracking of piezoelectric positioning stage using current-cycle iterative learning control with gain scheduling," IEEE Transactions on Industrial Electronics, vol. 61, no. 2, pp. 1085-1098, 2014.

[9] Q. Zhu, J.-X. Xu, D. Huang, and G.-D. Hu, "Iterative learning control design for linear discrete-time systems with multiple high-order internal models," Automatica, vol. 62, pp. 65-76, 2015.

[10] M. Sun and D. Wang, "Iterative learning control with initial rectifying action," Automatica, vol. 38, no. 7, pp. 1177-1182, 2002.

[11] J.-X. Xu and R. Yan, "On initial conditions in iterative learning control," IEEE Transactions on Automatic Control, vol. 50, no. 9, pp. 1349-1354, 2005. 
[12] R. Chi, Z. Hou, and J. Xu, "Adaptive ILC for a class of discretetime systems with iteration-varying trajectory and random initial condition," Automatica, vol. 44, no. 8, pp. 2207-2213, 2008.

[13] J.-X. $\mathrm{Xu}$ and J. $\mathrm{Xu}$, "On iterative learning from different tracking tasks in the presence of time-varying uncertainties," IEEE Transactions on Systems, Man, and Cybernetics Part B: Cybernetics, vol. 34, no. 1, pp. 589-597, 2004.

[14] H.-S. Ahn, K. L. Moore, and Y. Q. Chen, "Stability analysis of discrete-time iterative learning control systems with interval uncertainty," Automatica, vol. 43, no. 5, pp. 892-902, 2007.

[15] S. S. Saab, "A discrete-time learning control algorithm for a class of linear time-invariant systems," IEEE Transactions on Automatic Control, vol. 40, no. 6, pp. 1138-1142, 1995.

[16] C. Yin, J.-X. Xu, and Z. Hou, "A high-order internal model based iterative learning control scheme for nonlinear systems with time-iteration-varying parameters," IEEE Transactions on Automatic Control, vol. 55, no. 11, pp. 2665-2670, 2010.

[17] C. Liu, J. Xu, and J. Wu, "On iterative learning control with high-order internal models," International Journal of Adaptive Control and Signal Processing, vol. 24, no. 9, pp. 731-742, 2010.

[18] M. Yu, X. Ye, and D. Qi, "Repetitive learning control for triangular systems with unknown control directions," IET Control Theory \& Applications, vol. 5, no. 17, pp. 2045-2051, 2011.

[19] M. Yu, J. Wang, and D. Qi, "Discrete-time adaptive iterative learning control for high-order nonlinear systems with unknown control directions," International Journal of Control, vol. 86, no. 2, pp. 299-308, 2013.

[20] M. Yu, J. Wang, and D. Qi, "Feedback nonlinear discrete-time systems," International Journal of Systems Science, vol. 45, no. 11, pp. 2251-2259, 2014.

[21] M. Yu, D. Huang, and W. He, "Robust adaptive iterative learning control for discrete-time nonlinear systems with both parametric and nonparametric uncertainties," International Journal of Adaptive Control and Signal Processing, vol. 30, no. 7, pp. 972985, 2016.

[22] G. C. Goodwin, P. J. Ramadge, and P. E. Caines, "Discrete-time multivariable adaptive control," IEEE Transaction on Automatic Control, vol. 25, no. 3, pp. 449-456, 1980.

[23] W. Yan and M. Sun, "Adaptive iterative learning control of discrete-time varying systems with unknown control direction," International Journal of Adaptive Control and Signal Processing, vol. 27, no. 4, pp. 340-348, 2013.

[24] Q. Zhu, J.-X. Xu, S. Yang, and G.-D. Hu, "Adaptive backstepping repetitive learning control design for nonlinear discrete-time systems with periodic uncertainties," International Journal of Adaptive Control and Signal Processing, vol. 29, no. 4, pp. 524$535,2015$.

[25] Q. Zhu, G.-D. Hu, and W.-Q. Liu, "Iterative learning control design method for linear discrete-time uncertain systems with iteratively periodic factors," IET Control Theory \& Applications, vol. 9, no. 15, pp. 2305-2311, 2015. 


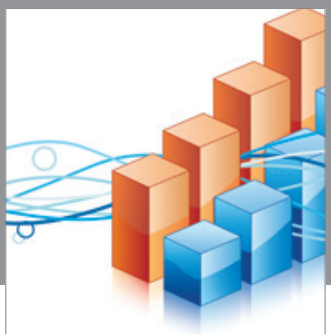

Advances in

Operations Research

vatem alat4

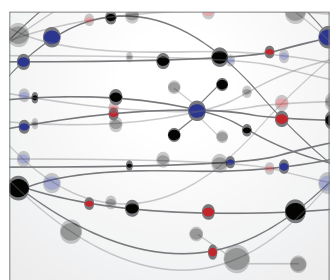

\section{The Scientific} World Journal
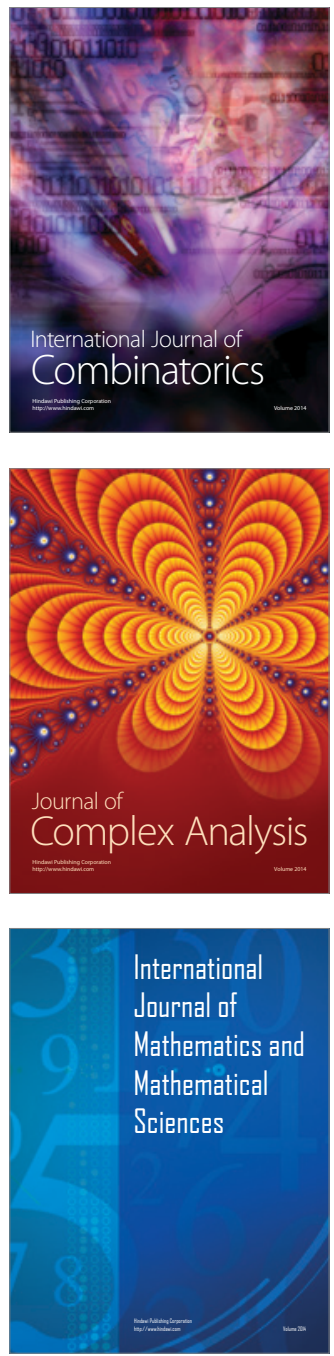
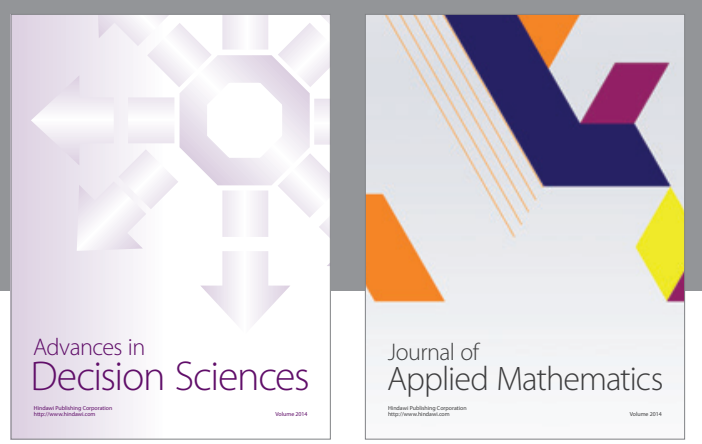

Algebra

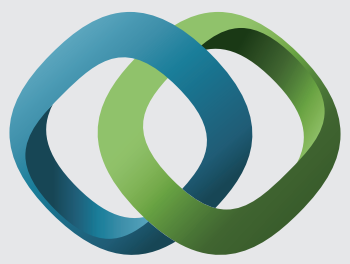

\section{Hindawi}

Submit your manuscripts at

http://www.hindawi.com
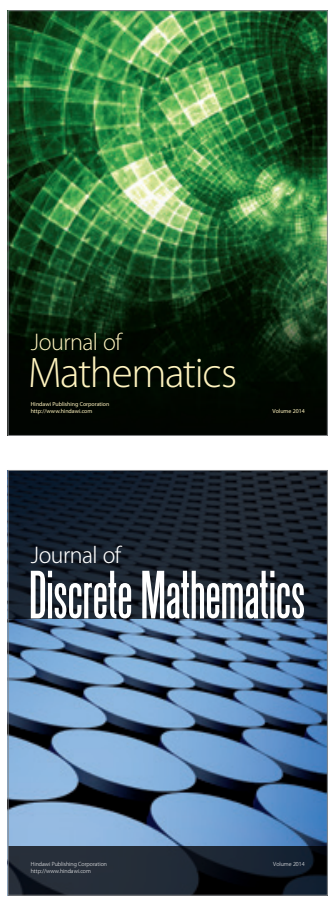

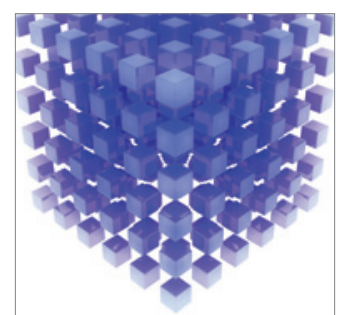

Mathematical Problems in Engineering
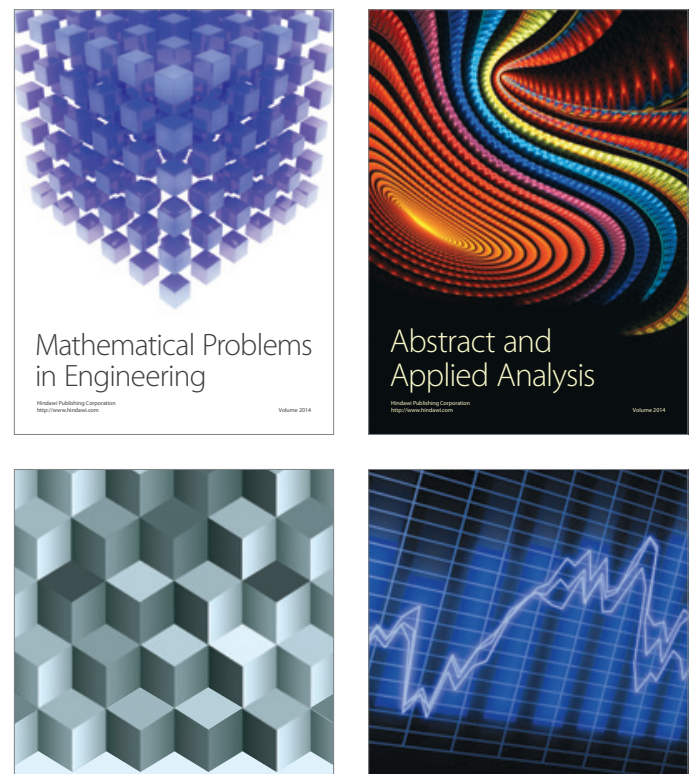

Journal of

Function Spaces

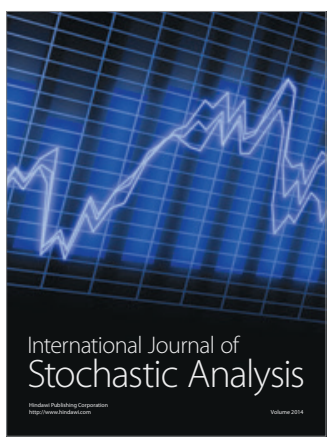

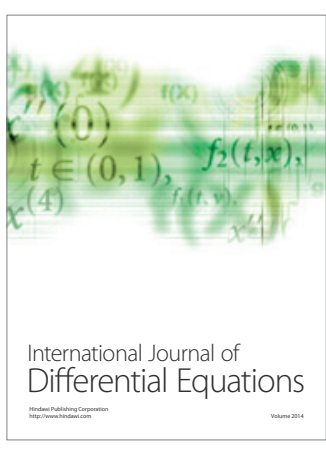
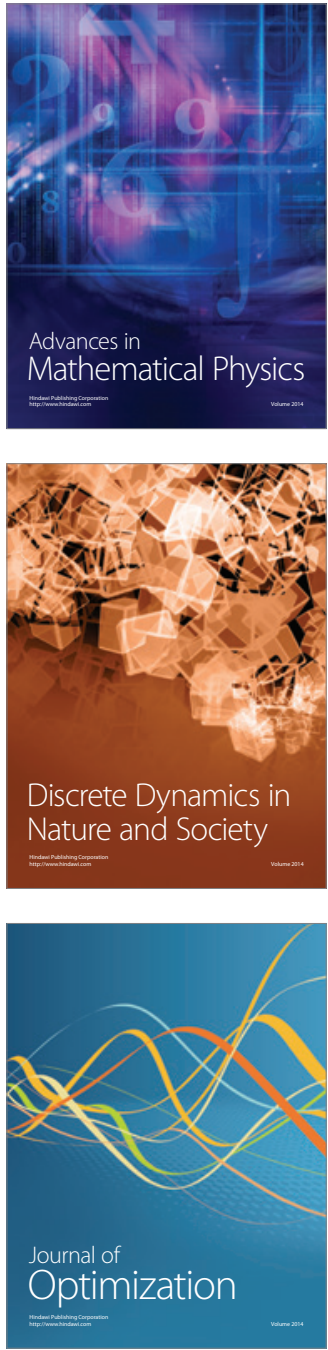